\title{
Heartbeat: Focus on hypertrophic cardiomyopathy
}

doi:10.1136/heartjnl-2016-310734

The focus of this issue of Heart is hypertrophic cardiomyopathy (HCM). As our ability to diagnosis HCM has improved due to the wide availability of genetic testing and advances in imaging, there has been increasing interest in the clinical presentation, outcomes and management of these patients.

Dynamic left ventricular (LV) outflow obstruction is a key feature of hypertrophic cardiomyopathy (HCM) yet the impact of relief of obstruction with alcohol septal ablation (ASA) on clinical outcomes remains unclear. In a series of 270 patients with HCM undergoing ASA, the outflow gradient was reduced to less than $30 \mathrm{~mm} \mathrm{Hg}$ in $77 \%$ of patients while $23 \%$ has persistent subaortic obstruction after the procedure (see page 1793). Persistent obstruction was associated with a higher cardiovascular mortality rate at 1 , 5 and 10 years of follow-up (figure 1 ). Multivariate predictors of cardiovascular mortality were persistent outflow obstruction early after hospital discharge (HR $2.95,95 \%$ CI 1.26 to $6.91 ; \mathrm{p}=0.01)$ and baseline septum thickness (HR 1.07, 95\% CI 1.01 to $1.13 ; \mathrm{p}=0.02$ ).

These findings are put into perspective in the editorial by Steggerd and ten Berg (see page 1775) which summarizes the previously published data on results with surgical meyctomy compared to ASA for HCM with subaortic obstruction. They note that most common adverse clinical outcome in the study by Veselka et al (see page 1793) was resuscitated sudden cardiac death. However, despite a higher risk of periprocedural ventricular arrhythmias with ASA compared to myectomy, possibly related to higher residual gradients, overall survival is similar with both procedures. ASA is associated with a higher rate of permanent pacer implantation, whereas hospital stay is longer with myectomy (table 1). Steggerd and ten Berg conclude "ASA is now a technique with a long history of research and improvement over time, which has led to the currently known excellent long-term survival and its effectiveness for improvement of symptoms." "Future improvements should provide performance of

Correspondence to Professor Catherine M Otto, Division of Cardiology, University of Washington, Seattle, WA 98195, USA; cmotto@u.washington.edu

Antonio Luiz P Ribeiro, Catherine M Otto

ASA with surgical precision in patients without unnecessarily increasing infarct size".

In another paper in this issue, Sheikh and colleagues (see page 1797) describe a cohort of 425 HCM patients from three British cardiomyopathy centres, followed for 4.3 years: 163 black and 262 Caucasian (white). Some presentation features were different between black and white HCM patients including a higher prevalence of hypertension $(58.3 \%$ versus $31.7 \%$ ) among black patients, who more frequently had an abnormal ECG at presentation. Repolarization abnormalities were more prevalent in black patients, defined as T-wave abnormities (91.4\% versus $73.0 \%$ ), including lateral $\mathrm{T}$-wave abnormities and deep T-wave inversion with ST segment depression. The pattern

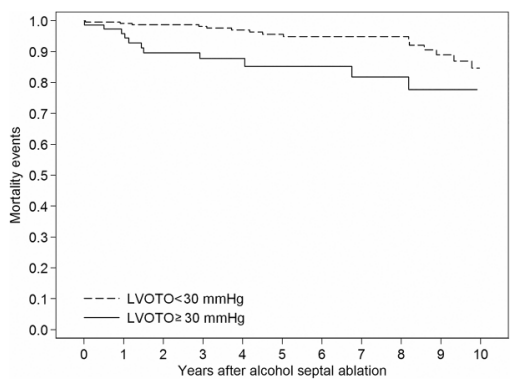

Figure 1 Kaplan-Meier curves describing freedom from cardiovascular mortality in patients with residual left ventricular outflow tract obstruction (LVOTO) $<30 \mathrm{~mm} \mathrm{Hg}$ and $\geq 30 \mathrm{~mm} \mathrm{Hg}$ (adjustment for age, sex, baseline LVOTO and baseline septum thickness). of LV hypertrophy in black, compared to white, patients was more often apical $(22.2 \%$ versus $10.7 \%)$ and concentric $(9.3 \%$ versus $1.5 \%)$ rather than asymmetric basal septal hypertrophy. Electrical and structural differences between black and white patients with HCM persisted even after excluding individuals who may be considered to have hypertensive heart disease, and may reflect the impact of ethnicity on the HCM phenotype. The authors conclude that a diagnosis of HCM should be considered in black patients with a history of hypertension who exhibit marked LV hypertrophy and T-wave inversion in the lateral leads despite good blood pressure control. These differences in phenotypic expression of HCM related to ethnicity did not impact the prognosis: HCM in black patients was associated with a similar prognosis as white patients, and hypertension and non-sustained ventricular tachycardia were the main prognostic factors in both groups (figure 2).

In the companion editorial, Spirito and Ferrazzi (see page 1777) point out that it is surprising how little is known about the impact of ethnicity on the clinical presentation and natural history of HCM in patients of African descent, which may reflect black patients having limited access to tertiary HCM referral institutions. They observed that the higher prevalence of hypertension in black patients may have led to over diagnosis of HCM, an issue addressed by Sheikh et al both in the analysis and the discussion. They conclude

Table 1 Current results of both procedures

\begin{tabular}{|c|c|c|c|}
\hline & Myectomy & ASA & $\mathrm{p}$ Value \\
\hline \multicolumn{4}{|l|}{ Efficacy/effectiveness } \\
\hline Symptomatic relief & $95 \%$ & $92 \%$ & 0.43 \\
\hline Gradient at follow-up ( $\mathrm{mm} \mathrm{Hg}$ ) & $12.2 \pm 3.8$ & $19.7 \pm 6.4$ & $<0.001$ \\
\hline Reintervention & $1.6 \%$ & $7.7 \%$ & $<0.001$ \\
\hline \multicolumn{4}{|l|}{ Complications } \\
\hline Periprocedural mortality & $1.1 \%$ & $1.3 \%$ & 0.75 \\
\hline Periprocedural VT/VF & $0.2 \%$ & $2.2 \%$ & 0.055 \\
\hline In-hospital stay (days) & $9(4-6)$ & $5(4-6)$ & $<0.001$ \\
\hline PM implantation & $4.0 \%$ & $10 \%$ & $<0.001$ \\
\hline \multicolumn{4}{|l|}{ Survival } \\
\hline Overall survival & $1.4 \% /$ year & $1.5 \% /$ year & 0.78 \\
\hline$S C D+I C D$ shock & $0.49 \% /$ year & $0.41 \% /$ year & 0.47 \\
\hline SCD-ICD shock* & $0.47 \% /$ year & $0.34 \% /$ year & 0.16 \\
\hline
\end{tabular}



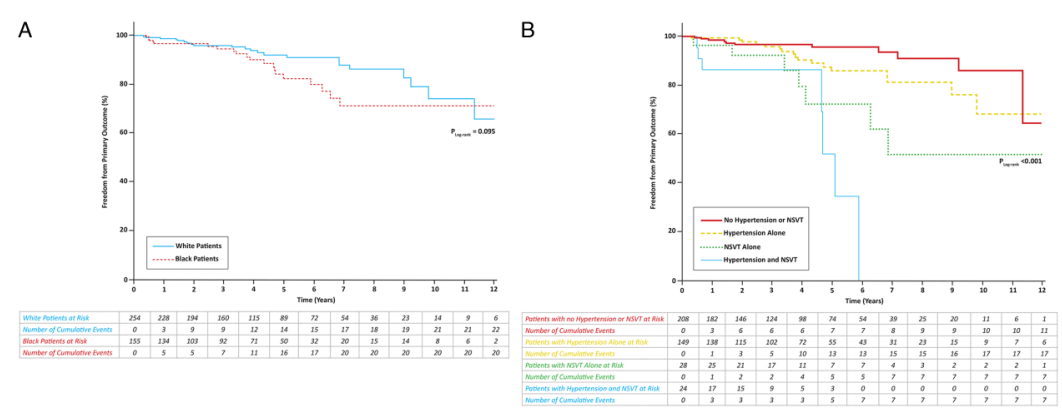

Figure 2 Kaplan-Meier curves illustrating freedom from the primary outcome during the study (A) according to ethnicity and (B) according to the presence or absence of NSVT and/or hypertension, in various combinations, for the overall cohort of black and white patients eligible for survival analysis $(\mathrm{n}=409)$. NSVT, non-sustained ventricular tachycardia.

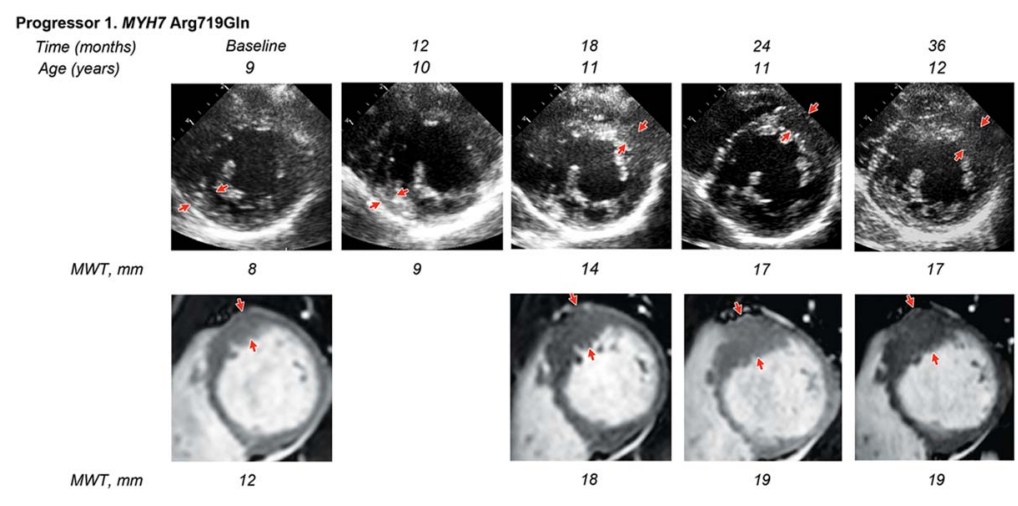

Figure 3 Serial echocardiographic and cardiac magnetic resonance (CMR) images in a participant who progressed to hypertrophic cardiomyopathy (HCM). Arrows indicate the region of maximal left ventricular (LV) wall thickness. Progressor 1: Female, 9 years of age at enrolment (myosin heavy chain (MYH7) mutation p.Arg719GIn)—randomised to diltiazem. Baseline echocardiogram suggested borderline focal septal hypertrophy that appeared more prominent on CMR (CMR maximal LV wall thickness of $12 \mathrm{~mm}$ involving one segment). Echocardiograms at 6 and 12 months of follow-up showed no definitive change. Echocardiography at 18 months showed an increase in basal septal hypertrophy to $14 \mathrm{~mm}$. CMR was performed at 18-month follow-up for careful reassessment of LV morphology and showed more prominent and extensive LV hypertrophy, with a maximal wall thickness of $18 \mathrm{~mm}$ now involving three segments. There was no late gadolinium enhancement. At 24 months, echocardiography showed localised hypertrophy, measuring up to $17 \mathrm{~mm}$ (z-score 10.9). CMR was unchanged from the 18-month study. At 36-month follow-up (1 year after stopping study medication), there was no significant change in LV wall thickness by echocardiography or CMR, and no visible late gadolinium enhancement.

that "the possibility of an HCM misdiagnosis in some blacks, rather than casting doubt on the results of the study, further underscores the complexity of reaching a proper HCM diagnosis in black patients, as well as the need to know more about the clinical presentation and outcome of HCM in this large ethnic group".

In an effort to elucidate the relationship between genotype and phenotype in HCM patents, Ho and colleagues (see page 1805) measured changes in LV geometry and function in 38 patients with sarcomere mutations who had no evidence of LV hypertrophy at baseline. Over a mean follow-up of 2.9 years, 4 patients $(11 \%)$ developed overt evidence of HCM (figure 3). Baseline predictors of progression to an HCM phenotype included a higher LV ejection fraction, lower early diastolic tissue velocity, higher $\mathrm{N}$ terminal pro peptide of B-type natriuretic peptide (NT-proBNP), larger posECG abnormalities. In addition, patients from families with highly penetrant mutations were more likely to have phenotypic progression over the short term. terior mitral leaflets, and more prevalent

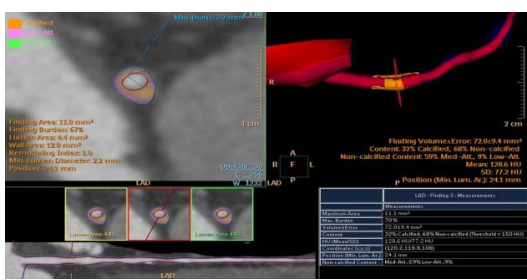

Figure 4 Semiautomated quantification of luminal area and plaque volume obtained from a patient with mild to moderate obstructive left anterior descending coronary stenosis.

In an editorial, Captur and Moon (see page 1779) comment that "We, like Ho et al, are of the belief that large-scale research efforts focusing on the systematic, high-resolution and multimodality scrutiny of the early (subclinical) HCM phenotype may offer superior genotypephenotype insights into prognosis and response to therapy". This editorial also includes a summary table that lists the morphostructural abnormalities seen in patients with subclinical HCM, including cut-off values for identification of affected individuals.

The Education in Heart article in this issue (see page 1840) provides an introduction to cardiac computed tomography (CCT). This modality offers high resolution imaging of coronary anatomy, including the degree and location of calcification, direct visualization of the coronary vessel wall, and functional imaging for ischemia with hybrid approaches (figure 4). CCT evaluation of cardiac structure and function is increasingly important in management of patients with aortic disease, adult congenital heart disease, and valvular heart disease.

The Image Challenge (see page 1839) in this issue shows an unusual hourglass appearance on ventriculography as well as on cardiac magnetic resonance images. An interesting differential diagnosis with intriguing online videos to supplement the still images.

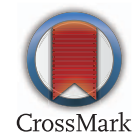

To cite Ribeiro ALP, Otto CM. Heart 2016;102:1773-1774.

Heart 2016:102:1773-1774.

doi:10.1136/heartjnl-2016-310734 